\title{
LA CONSTRUCCIÓN DE ESCENARIOS DE FUTURO COMO HERRAMIENTA PARA LA EDUCACIÓN AMBIENTAL EN EDUCACIÓN SECUNDARIA OBLIGATORIA
}

\author{
Juan José Ochando López \\ Colegio Nuestra Señora de la Salud (Xirivella, España) \\ Ignacio García Ferrandis ${ }^{1}$ \\ Universidad de Valencia. Valencia, España \\ Javier García Gómez \\ Universidad de Valencia. Valencia, España
}

\begin{abstract}
Resumen. En el ámbito escolar se hace referencia al futuro profesional del alumnado, sin embargo, no siempre se habilitan espacios para la reflexión sobre el futuro de una manera atractiva. La presente investigación pretende conocer en qué medida el alumnado de Educación Secundaria Obligatoria deja patente lo aprendido en materia de Educación Ambiental para la Sostenibilidad mediante la reflexión y proyección de su propio futuro a través la Construcción de Escenarios de Futuro (C.E.F.). Esta herramienta puede servir para la reflexión del alumnado sobre el futuro probable y el deseable o posible, y tome conciencia de su papel como parte activa en la construcción de ese futuro y la necesidad de su formación para ello. El instrumento de toma de datos fue la narrativa, con carácter de escrito de producción propia por parte del alumnado. Con las respuestas del alumnado se hizo un análisis de contenido para lo que se tomó como base la enumeración de términos propios a las temáticas curriculares en materia de Educación Ambiental. La C.E.F. puede ayudar a conocer lo que hasta el momento ha sido significativo y asimilado por el alumnado, además permite analizar y comprender los intereses, miedos, y fortalezas percibidas por ellos ante su futuro probable generado. A raíz del interés mostrado, la C.E.F. puede resultar una herramienta docente eficaz para la determinación y programación de Proyectos Ambientales interdisciplinares destinados a mejorar las condiciones de la realidad en la que el alumnado están viviendo.
\end{abstract}

Palabras clave: Educación Ambiental, Construcción de Escenarios de Futuro, Educación Secundaria Obligatoria.

\section{THE CONSTRUCTION OF FUTURE SCENARIOS AS A TOOL FOR ENVIRONMENTAL EDUCATION IN COMPULSORY SECONDARY EDUCATION}

\begin{abstract}
The professional future of students is addressed within school settings. However, not always spaces for reflection about the future in an attractive way, are enabled. The present research aims to find out what students of secondary education have learned in the Environmental Education for sustainability course through the reflection and projection of their own future through the Construction of Future Scenarios (C.E.F.). The C.E.F. can serve for student's reflection about their likely, desirable and possible

${ }^{1}$ Ignacio García Ferrandis. Avda. Tarongers no 4, 46022 València. ignacio.garcia-ferrandis@ uv.es
\end{abstract}


future. It also serves to make them aware about their own role as an active agent in the construction of that future and the need for academic qualification to do so. The instrument for data collection was the narrative, so students writing production was analyzed. We carried out a content analysis of their responses, coding specific terms and topics within the field of environmental education. The C.E.F. can help us to know what has been significant and assimilated by the students so far. It also allows us to analyze and understand the interests, fears, and strengths they perceived around its likely future. The results show that C.E.F. can be an effective teaching tool for setting and programming interdisciplinary environmental projects aimed to improve the conditions of students' reality.

Keywords: Environmental Education, Construction of Future Scenarios, Compulsory Secondary Education.

\title{
A CONSTRUÇÃO DE CENÁRIOS FUTUROS COMO UMA FERRAMENTA PARA A EDUCAÇÃO AMBIENTAL NO ENSINO SECUNDÁRIO OBRIGATÓRIO
}

\begin{abstract}
Resumo. No âmbito escolar são feitas referências ao futuro profissional dos nossos alunos. Entretanto, nem sempre são habilitados espaços para a reflexão sobre o futuro de um modo atrativo. O presente estudo teve como propósito conhecer em que medida os alunos do Ensino Secundário Obrigatório se apropriaram de conteúdos e conceitos envolvidos na Educação Ambiental para a Sustentabilidade, mediante a reflexão e a projeção do próprio futuro, expressadas na Construção de Cenários de Futuro (C.E.F.). O objetivo é que a C.E.F. possa servir para a reflexão sobre o futuro provável e o futuro desejável ou possível e, com isso, os alunos tomem consciência do seu papel como parte ativa na construção do futuro e a necessidade da sua formação para tal. A técnica de coleta de dados foi a narrativa, considerando a escrita de produção dos próprios alunos. A partir de suas respostas foi realizada uma análise de conteúdo, tomando como base a enumeração de termos referentes às temáticas curriculares relacionadas à Educação Ambiental. A C.E.F. pode nos ajudar a conhecer o que, até o presente momento, foi significativo e apropriado pelos alunos, além de possibilitar a análise e compreensão dos interesses, medos e qualidades percebidas por eles diante do seu futuro provável gerado. Devido ao interesse demonstrado, a C.E.F pode resultar em uma ferramenta docente eficaz para a determinação e programação de Projetos Ambientais interdisciplinares destinados a melhorar as condições da realidade que os alunos estão vivendo.
\end{abstract}

Palavras-chave: Educação Ambiental, Construção de Cenários de Futuro, Ensino Secundário Obrigatório.

\section{Introducción y Objetivo de la investigación}

Constantemente en el ámbito escolar se hace referencia al futuro profesional del alumnado, dentro de su ciudad, sus anhelos, sueños y deseos, y no menos veces se les indica que es, en el presente que viven donde empiezan a forjar su futuro. Pero, ¿les habilitamos verdaderos espacios en clase para la reflexión sobre el futuro de una manera atractiva y a su vez aplicada a la educación de la que son protagonistas? ¿Es posible hacer esto dentro de un currículo académico cargado de contenidos y muchas veces alejado de la realidad y vida del alumnado? 
Ante esta pregunta surge la idea de que el alumnado trabaje la Construcción de Escenarios de Futuro (C.E.F.), ya que puede resultar una herramienta eficaz en el aula para promover una conciencia sostenible y global, creando además en ellos la intencionalidad de cambiar sus conductas en pos de lograr el futuro proyectado por ellos mismos, y sirviendo de línea argumental en el desarrollo curricular de las asignaturas tratadas en el aula, en especial y como es objeto del presente estudio, en lo que a la Educación Ambiental dentro del espacio clase se refiere.

Desde las inquietudes anteriormente mencionadas, nace la consideración de que la Educación Ambiental puede valerse de los beneficios de la Construcción de Escenarios de Futuro para trabajar algunas de las indicaciones de la UNESCO en materia de Desarrollo Sostenible. Ésta organización en el 2006 indicaba que para alcanzar los objetivos marcados en el Decenio de la Educación para el Desarrollo Sostenible, sería necesario reorientar los programas educativos, de manera que las sociedades futuras fueran educadas en unos conocimientos, actitudes, perspectivas y valores relacionados con la sostenibilidad. Por otro lado, propuso que se debía integrar en el aprendizaje los aspectos esenciales del desarrollo sostenible, cambio climático y reducción de la pobreza entre otros.

A su vez Matsuura (UNESCO, 2006), Director General de la UNESCO, indicaba que la educación, en todas sus formas y todos sus niveles, no es sólo un fin en sí mismo, sino también uno de los instrumentos más poderosos con que contamos para inducir los cambios necesario para lograr un desarrollo sostenible. Como se extrae de las indicaciones de la UNESCO, se puede concretar el interés del presente estudio en aras de buscar la reorientación de los programas educativos y el uso del ámbito educativo para promover en el alumnado una actitud activa dentro del desarrollo sostenible.

En la presente investigación se pretende conocer si el alumnado de Educación Secundaria Obligatoria en capaz de movilizar sus conocimientos en materia de Educación Ambiental. Para ello se les propone una actividad en la que trabajan mediante la Construcción de Escenarios de Futuro, de manera que se puedan observar dos cuestiones básicas como son, la cantidad de conceptos adquiridos en materia de Educación Ambiental, y las relaciones causa-efecto que perciben en el futuro. De esto modo se supone que se podrán deducir los problemas percibidos como más relevantes por el alumnado, y en un futuro reorientar la práctica docente tal y como indica la UNESCO, afrontando el curriculum como un medio para avanzar o modificar el futuro previsto.

\section{Educación Ambiental y la Construcción de Escenarios de Futuro}

La Educación Ambiental (E.A.) puede incorporarse al currículum de diferentes modos, y grados de intensidad, bien como un recurso didáctico más a utilizar dentro del aula para la consecución de diferentes objetivos o capacidades del alumnado, o como un 
principio didáctico que impregna diferentes asignaturas y contenidos curriculares, de manera que actúa como eje vertebrador en el aprendizaje (Sessano, 2006).

La integración de la Educación Ambiental en la Educación Secundaria Obligatoria sigue teniendo dificultades para su incorporación efectiva en el aula. El profesorado reconoce que los numerosos problemas de adaptación del sistema a los planteamientos de la reforma educativa, la falta de los suficientes apoyos externos y los problemas no resueltos de gestión interna de los centros, llevan a una incorporación deficiente de la Educación Ambiental dentro de las instituciones educativas con toda su potencialidad (Pascual et al., 2000).

En este sentido, la incorporación de la Educación Ambiental en Educación Secundaria Obligatoria en Ciencias Naturales, Sociales o Tecnología, o de manera transversal en otras áreas ha supuesto, desde hace años, el reconocimiento desde el sistema educativo y el currículum al importante papel que juega el medio ambiente en la vida de las personas y en el desarrollo de la sociedad (Muñoz, 2010). Aun así, se sigue estando lejos de las pretensiones anteriormente comentadas por la UNESCO al respecto de la reorientación de los programas educativos quedando además pendiente, la adquisición de las capacidades que permitan al alumnado llevar a término lo especificado en el Congreso Internacional de Educación y Formación sobre Ambiente celebrado en Moscú en 1987, que indicaba que la Educación Ambiental es un proceso permanente en el cual los individuos y las comunidades adquieren consciencia de su ambiente, aprenden los conocimientos, los valores, las destrezas, la experiencia y también la determinación que les capacite para actuar, individual y colectivamente, en la resolución de los problemas ambientales presentes y futuros.

Por todo lo anteriormente citado, es necesario destacar que, la Educación Ambiental no es un simple conjunto de conocimientos, sino una actitud creativa y práctica diaria hacia la comprensión y transformación del proceso histórico, su ubicación en el cosmos, el pensamiento humano y su nivel socio-económico y relación ambiental; pues avanza a través de la solución a los nuevos paradigmas humanísticos que buscan evolucionar y adaptarse a las nuevas circunstancias y problemas actuales, como el deterioro ambiental y social (Novo, 2006).

La Educación Ambiental es el ámbito científico que alimenta esta investigación, pero como aspecto novedoso utiliza la Construcción de los Escenarios de Futuro, dentro de la labor docente para, como se ha indicado con anterioridad, observar tanto la cantidad de conocimientos en E.A. adquiridos por el alumnado, como las relaciones causa-efecto percibida por el mismo. De modo pretendemos que la formación recibida por el alumnado, sea vista por éste, como un medio de transformación hacia el futuro deseado.

El modelo educativo ha de ir orientado a la reflexión sobre el futuro del alumnado y la C.E.F. puede servir para este propósito. A nivel general y sobre todo en el ámbito de la planificación y gestión, si se quiere reflexionar, analizar y predecir el 
futuro, se pueden utilizar diversas técnicas como modelos causales, análisis prospectivos, simulaciones, pronósticos cualitativos, cuantitativos, entre otros, teniendo siempre como objetivo determinar los posibles problemas y obstáculos futuros así como las posibles oportunidades para llevar a cabo acciones presentes que nos conduzcan al final deseado. En esta investigación hemos optado por la C.E.F. A continuación, se explica brevemente su definición, evolución y aplicaciones para finalmente ver su potencialidad como herramienta para trabajar en el aula.

La planeación por escenarios (o análisis de escenarios) tiene su origen en los años 40 del siglo pasado, pero se consolida como un modelo de planeación organizacional en 1950. Introducido por Herman Kahn, quien trabajaba para el Departamento de Defensa de los Estados Unidos, lo popularizó diez años después como director del Hudson Institute (EE.UU.). En 1967, en compañía de Anthony Wiener, publicó "The year 2000", donde establece una serie de escenarios mundiales para un periodo de proyección de 33 años. Desde entonces, se han sucedido diversos modelos como los de Dubin, Lynham y Van de Ven, siendo el propuesto por Dubin el más influyente, sobre todo dentro del ámbito empresarial donde la C.E.F. ha tenido su mayor desarrollo (Vergara et al., 2010).

De las diversas definiciones de C.E.F. existentes, las más clarificadoras dentro del presente estudio son las de Khan y Wiener (1967) y Kliener (1999). Según los primeros autores, se considera los escenarios, como secuencias hipotéticas de eventos construidos con el propósito de centrar la atención en los procesos causales y la toma de decisiones. Sin embargo, para el último autor, los escenarios son cuadros o pinturas imaginadas sobre futuros potenciales.

A nivel empresarial existen diferentes modelos de trabajo para la construcción de escenarios de futuro, como los propuestos por Wack (1985) de tipos cualitativos y cuantitativos; Schwartz (1996) con una metodología de ocho pasos entre los cuales se tienen en cuenta tanto aspectos como el pensamiento crítico o la generación de futuros posibles de acuerdo a las implicaciones de las decisiones de factores múltiples, entre otros; o Porter (2004) que se basa en el análisis del sector industrial.

En 2010, Vergara et al., ya planteaba que aunque la planeación de escenarios era un área de estudio ligada a la planeación estratégica, existía un gran interés por explotar esta herramienta en el mundo empresarial, pero también y especialmente en el académico

Por lo anterior, se considera que la C.E.F. puede ser el medio donde el alumnado podrá poner en marcha todos sus mecanismos creativos para construir un posible futuro mediante sus apreciaciones pero también mediante los conocimientos aprendidos durante su vida, tanto a nivel formal como no formal. Para la Construcción de los Escenarios de Futuro es necesario poner en movimiento e interrelacionar todo lo aprendido. Mediante el uso de la C.E.F. como herramienta se pretende que el alumnado a través de su conocimiento adquirido a nivel curricular, su propia experiencia vital y su 
creatividad sea capaz de describir el escenario futuro en el que perciba que va a tener que vivir. Esta herramienta basada en la prospectiva, permite que el alumnado reflexione sobre el futuro probable y el futuro deseable o posible, y tome conciencia de su papel como parte activa en la construcción de ese futuro y la necesidad de su formación para ello.

La Construcción de Escenarios de Futuro, no solo permite saber lo que hasta el momento ha sido significativo y adquirido por el alumnado sino que permite analizar y comprender los intereses, miedos, y fortalezas percibidas por ellos ante su futuro probable generado. Los C.E.F. pueden servir como herramienta para, sin obviar los contenidos curriculares de la ley, estructurar "Qué" y "Cuándo" afrontar el temario dándole vida a la programación de aula, muchas veces un documento encorsetado y estático.

La C.E.F. se apoya en tres postulados que tienen especial importancia en el ámbito educativo como son la libertad, el poder y la decisión. Lo que es de especial importancia ya que es necesario que los estudiantes utilicen la C.E.F. como una herramienta que les permita descubrir que el futuro no está escrito, ni determinado, sino que está abierto a una multitud de futuros posibles. Si no se produce este paso y conocimiento del estudiante del concepto de libertad, la C.E.F. puede quedarse en una mera redacción o conjunto de actividades sin implicación directa en su vida.

Además se ha de hacer sentir al estudiante que tiene "el poder" de intervenir directamente en el devenir de los acontecimientos de la historia que le ha tocado vivir, sin necesidad de dejarse llevar por la corriente o lo mayoritariamente aceptado. Esto presenta un especial conflicto en los adolescentes que si bien, presentan unas grandes ansias de cambiar el mundo que les rodea, su visión y perspectivas de futuro se centran en la inmediatez y el corto plazo, por lo que la C.E.F. debería de trabajar con anterioridad en el fortalecimiento de la constancia y perseverancia como actitudes necesarias para un verdadero cambio de su mundo e historia.

Por otro lado, la C.E.F. puede quedarse en una simple exposición de sueños y deseos si no se aterriza a la realidad desde el primer día. Es aquí donde metodologías muy utilizadas en otro países (incluso obligatorias dentro del currículum escolar) y en España desde hace algunos años en unos pocos lugares, como son las asociadas al Aprendizaje-Servicio podrían ser el complemento ideal a las C.E.F. en la formación de estudiantes comprometidos con su proceso enseñanza-aprendizaje y como ciudadanos de acción transformadora del mundo que les rodea.

Normalmente el desarrollo de los Escenarios de Futuro es un instrumento utilizado en los ámbitos políticos y económicos. En el ámbito educativo son pocas las referencias bibliográficas que analizan las visiones del futuro en el aula, cabe citar a Kong (2002) o el estudio sobre las ilusiones y miedos de los niños y niñas de Pamplona ante el futuro realizado por las autoras Reparaz y Naval (2007). 


\section{Método}

Para dar respuesta al propósito de la presente investigación y analizar las representaciones de los imaginarios futuros de un grupo de estudiantes, en el marco de la Educación Ambiental, se trabajó desde el enfoque interpretativo. La toma de datos se realizó mediante la técnica de la narrativa y posteriormente, para el estudio de los datos se utilizó el análisis de contenido. El presente estudio es de corte descriptivo.

El término investigación interpretativa se refiere a toda una familia de enfoques de investigación participativa observacional. Se utiliza a modo de reemplazar el término investigación cualitativa, por uno más incluyente, que nos permita evitar la idea de que sea esencialmente no cuantitativo y apunte al interés central de esta investigación que es el significado humano en su contexto social y su dilucidación y exposición por el investigador (Erickson, 1986).

Por tanto, la presente investigación se enmarca dentro del paradigma interpretativo con el foco de interés en la Construcción de Escenario de Futuro. Como indica Kong (2014, p. 86), "el paradigma interpretativo busca explicitar los significados subjetivos de las acciones de los actores sociales, descubrir lo que da sentido a las actividades sociales, para así develar la estructura de inteligibilidad que da cuenta por qué dichas acciones tienen sentido para los sujetos". En esta investigación es fundamental captar la realidad prospectiva y socioeducativa de los sujetos actuantes a partir de la percepción que tienen de su propio contexto y visión de futuro. Ésta última se basa en nuestras creencias, condiciones, actitudes, deseos e historia de vida personal.

\section{Muestra e Instrumento}

El presente estudio se ha realizado en el colegio concertado de la localidad de Xirivella, a escasos kilómetros de la ciudad de Valencia. El alumnado objeto de estudio ha sido los integrantes de $1^{\circ}$ E.S.O (31 alumnos/as), $2^{\circ}$ E.S.O (32 alumnos/as), $3^{\circ}$ E.S.O (22 alumnos/as) y $4^{\circ}$ E.S.O (16 alumnos/as). Se ha elegido este tipo de colegio, por las facilidades para realizar la investigación ya que uno de los autores desarrolla su labor docente en el mismo. Todos los estudiantes de las muestras pertenecían a grupos naturales en sus centros educativos. Se trató de muestras de conveniencia a las que se tuvieron acceso tras obtener los permisos pertinentes. En todo caso, los estudiantes no sufrieron selección alguna para ser asignados a los grupos naturales.

El instrumento para la toma de datos utilizado fue la narrativa. La investigación narrativa engloba distintos modos de obtener y analizar relatos referidos a experiencias personales, que tendrán en común la reflexión (oral o escrita) que utiliza la experiencia personal en su dimensión temporal. La investigación narrativa ha adquirido cierta relevancia en las ciencias sociales y humanas en las últimas décadas. Según Polkinghorne (1995, p. 5), "la narración es la única forma lingüística adecuada para mostrar la existencia humana como acción contextualizada". 
En el contexto español cabe destacar la aportación de Rivas, Herrera y Kushner (2009) en la investigación biográfica-narrativa en educación y de Hernández (2004) que hace una síntesis sobre la situación de este tipo de investigación en España. Como indican los primeros, la narrativa es una perspectiva metodológica con una trayectoria breve y no siempre bien comprendida en el ámbito educativo, si bien hay ciertos indicios que invitan al optimismo.

Al igual que Jiménez (2006), opinamos que las narrativas expresan un modo de contar historias sobre nuestras vivencias y proyecciones, reflejan una visión del mundo y una construcción particular de la realidad, concibiendo así la narración como un producto lingüístico y discursivo que tiene una organización interna propia. Por otro lado, somos conscientes que la investigación narrativa es en cierto modo diferente en las ciencias sociales y en particular en educación, que pretende hacer frente a las corrientes positivistas en su intento de segregar al sujeto del investigador.

En cierta medida, el interés de esta investigación reside en su aspecto novedoso. Como indican Rivas, Herrera y Kushner (2009), la mayoría de la literatura en relación al estudio de las biografías en el ámbito educativo, se limitan al profesorado. En el caso del alumnado o de otros componentes de la comunidad educativa, resulta prácticamente inexistente. En el caso de los estudiantes, cabe mencionar los trabajos de Suárez Pazos (2002 y 2004) sobre memorias escolares y Calderón y Garrido, (2004) sobre alumnado en situación de fracaso.

Referido a las materias, el uso de las narrativas y las biografías en la enseñanza de las distintas disciplinas del currículum, se ha centrado especialmente en Inglés e Historia. Por este motivo, esta investigación supone también una novedad al trabajar aspectos relacionados con la Educación Ambiental. En nuestro caso, las narraciones realizadas por el alumnado muestran su visión del futuro desde un punto de vista global respecto de su propia vida. Para proyectar el futuro es necesario crear desde la propia experiencia obtenida en cualquier campo de la vida cotidiana, en este caso, de manera fundamental a nivel curricular.

Tal y como indica Gibbs (2012) el análisis de la narrativa no se centra exclusivamente en qué se dice sino también en cómo se dice. En este sentido, Rivas (en Rivas, Herrera y Kushner, 2009, p.18) presenta cómo la investigación narrativa supone una forma de conocimiento que interpreta la realidad desde una óptica particular: la de la identidad como una forma de aprendizaje de los contextos en los que los sujetos viven y los modos como los narramos en un intento de explicarnos el mundo en que vivimos. De este modo los sujetos de investigación pueden tener voz, y el investigador puede comprender y experimentar su mundo, en este caso su manera de ver y entender el futuro de acuerdo a los conocimientos y sentimientos que tienen en ese momento.

Por otro lado, Kong (2002) sostiene que la narración representa un modo de ver la realidad, sirve de puente para aproximarnos a pensamientos, crear e imaginar mundos, muchos de estos ficticios, pero con pretensiones de ser reales. Dicha realidad 
está influenciada por la información y la propia experiencia. A su vez, según el mismo autor, la narración nos permite comunicarnos y explicar nuestras visiones o representaciones del mundo. En cualquier caso, como indica Rivas (en Rivas, Herrera y Kushner, 2009, p.28), “el modelo de investigación es dinámico, abierto y vivo, por tanto requiere constantemente de una revisión personal y epistemológica sobre los principios que lo sustentan".

Con las narraciones ya recopiladas, la técnica para su estudio fue el análisis de contenido. El objetivo de esta técnica es estudiar, conocer y comprender qué tipos de contenidos manifiestos existen en una comunicación escrita clasificando sus diferentes partes de acuerdo con unas categorías previamente establecidas por lo que existe la posibilidad de identificar de manera sistemática y objetiva dichas categorías dentro de un mensaje.

Piñuel (2002) define el análisis de contenido como el conjunto de las interpretaciones que el investigador realiza sobre productos comunicativos tales como mensajes, textos, discursos, y que mediante técnicas de medida cuantitativas y cualitativas elaboran y procesan los datos para alcanzar un nuevo conocimiento o diagnóstico. La proliferación de estudios realizados con esta técnica, durante el siglo XX se afianzó y corroboró como una de las técnicas más utilizadas en estudios de ciencias sociales.

Cabe destacar que el sentido del contenido que se pretende analizar sólo puede ser captado dentro de un contexto, el cual es el marco de referencia poseedor de la información susceptible de conocer de antemano o que puede ser inferida a partir del propio texto, para captar el contenido y significado de lo que se dice. Por lo tanto, texto y contexto son dos aspectos fundamentales del análisis de contenido (López-Aranguren, 2015).

\section{Procedimiento}

Tras la determinación de los objetivos del estudio, y la elección del centro educativo en el que realizar el estudio, se procedió a aplicar la C.E.F. en cada uno de los cuatro cursos de la E.S.O. objeto de análisis, con la siguiente secuencia procedimental.

En primer lugar, se informó a los estudiantes del hecho de que iban a formar parte de un estudio para la Universidad de Valencia, pero sin desvelar la temática del mismo. El objetivo de este primer paso, fue situar al alumnado dentro del contexto de una investigación para lograr no solo su máxima colaboración, sino también su mayor grado de esfuerzo en la participación, ya que movilizar sus conocimientos o pedir una producción escrita en el tipo de centro educativo con las características socioeconómicas mencionadas suele ser problemático, si el alumnado no cuenta con la motivación suficiente.

A continuación, durante la misma sesión de clase, se solicitó al alumnado que desarrollasen una narrativa escrita en forma de carta con carácter anónimo. En ella 
debían contar cómo imaginaban el futuro. La pregunta que se planteó para generar cierta motivación para la narrativa fue la siguiente:

¿Cómo crees que será el mundo dentro de 20 años, cómo será tu vida en él, a qué te dedicarás?

Tras la realización de las producciones escritas, a cada una se le asignó un código en la cabecera compuesto, por un número-curso-hombre/mujer, para la correcta identificación del tipo de alumno/a estudiado, a pesar de ser anónimo. Seguidamente, con las respuestas del alumnado se procedió al análisis de contenido.

Como indica Álvarez-Gayou (2003), una característica de esta técnica es que el examen de los datos se realiza mediante codificación, por ésta se detectan los elementos relevantes del discurso. En nuestro caso, para determinar la unidad de registro del análisis de contenido se tomó como base los términos propios a las temáticas curriculares en materia de Educación Ambiental.

Para poder realizar el análisis de contenido, en primer lugar se analizaron los contenidos de cada curso presentes en el currículum de Educación Secundaria Obligatoria de la Comunidad Valenciana bajo el que han estudiado el alumnado objeto de estudio (tabla 1), con el objetivo de obtener los temas asociados que el alumnado debería de conocer según el curso en el que se encuentre. Una vez obtenidos los temas asociados a nivel de Educación Ambiental en cada uno de los cursos, se realizó la lectura de las respuestas del alumnado, seleccionando las palabras o frases que hacían referencia a conceptos medioambientales propios del currículum de Educación Secundaria Obligatoria.

Tabla 1: Asignaturas y temas relacionados con la Educación Ambiental del currículum de Educación Secundaria obligatoria por curso

\begin{tabular}{|c|c|c|}
\hline Curso & Asignaturas relacionadas $E . A$. & Temas asociados \\
\hline $\begin{array}{c}1^{\mathrm{o}} \\
\text { E.S.O }\end{array}$ & $\begin{array}{l}\text { Ciencias Naturales, Ciencias } \\
\text { Sociales y Tecnología }\end{array}$ & $\begin{array}{l}\text { Contaminación atmosférica e hídrica, explotación de } \\
\text { recursos metálicos y forestales, los riesgos climáticos, los } \\
\text { espacios naturales y los riesgos y políticas correctoras }\end{array}$ \\
\hline $\begin{array}{c}2^{\circ} \\
\text { E.S.O }\end{array}$ & Ciencias Naturales & $\begin{array}{l}\text { Energías renovables, el ahorro energético y los problemas en } \\
\text { la distribución la obtención, transporte y uso de la energía. }\end{array}$ \\
\hline $\begin{array}{c}3^{\circ} \\
\text { E.S.O }\end{array}$ & $\begin{array}{l}\text { Física y Química, Tecnología y } \\
\text { Ciencias Sociales }\end{array}$ & $\begin{array}{l}\text { Fuentes de energía, sus impactos y políticas correctoras, el } \\
\text { impacto de la globalización, y el consumo irracional, así } \\
\text { como la gestión sostenible de los recursos, la afección de la } \\
\text { química al medioambiente. }\end{array}$ \\
\hline $\begin{array}{l}4^{\circ} \\
\text { E.S.O. }\end{array}$ & $\begin{array}{l}\text { Física y Química y Biología y } \\
\text { Geología }\end{array}$ & $\begin{array}{l}\text { Concepto de Desarrollo Sostenible, y la globalización de la } \\
\text { contaminación y de los impactos ambientales que afectan } \\
\text { directamente a la biodiversidad (transgénicos, incendios } \\
\text { forestales, antropización incontrolada) }\end{array}$ \\
\hline
\end{tabular}

Fuente: Propia

En la figura 1 se presenta un ejemplo del procedimiento metodológico empleado sobre un texto de una alumna, observándose la codificación en cabecera, así como la 
detección de las unidades de contenido referidas a la educación ambiental. En este caso, la alumna mostraba especial preocupación por el calentamiento global, y pérdida de biodiversidad. Del mismo modo, se procedió con los escritos de todos los alumnos y alumnas elaborándose tablas con los códigos de las unidades de contenido que hacían referencia a los conceptos curriculares propios de la etapa educativa, así como el número de citas.

Finalmente, estos códigos junto a los detectados en el resto de escritos, se introdujeron en la aplicación Wordle-Create para generar una nube de palabras para cada curso, como se verá más adelante. Si bien las nubes de palabras no son un método de análisis en sí mismo, si son un método de presentación de resultados de fácil percepción, no solo de las temáticas destacadas sino a la relación de magnitud respecto de las demás.

Figura 1: Ejemplo de Análisis de Contenido aplicado a una actividad de Construcción de Escenario de Futuro. Alumna de $3^{\circ}$ E.S.O.

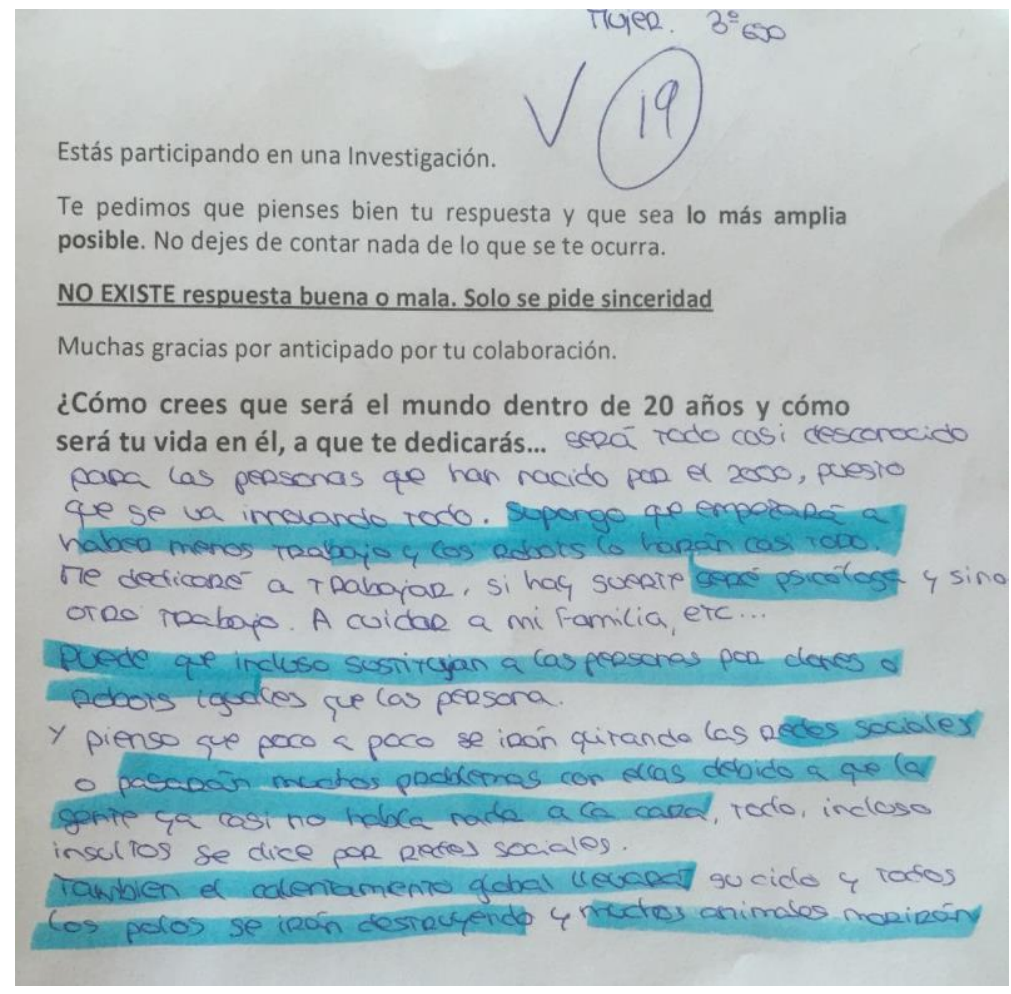

Fuente: Propia

\section{Resultados}

Los resultados del análisis de textos realizados se recogen en la tabla 2, clasificados por temáticas ambientales tratadas en los escritos del alumnado de los cuatro cursos de Educación Secundaria Obligatoria. En dicha tabla se puedan observar el número de citas para cada una de las temáticas propias del currículum, según los códigos aplicados elegidos tras el análisis de contenido realizado en cado uno de los cursos. En la primera columna se incluyen los contenidos curriculares de cada nivel, en la segunda los 
diferentes códigos asociados que han aparecido durante el estudio, y en las cuatro últimas el número de citas según el curso estudiado. A continuación se muestran los resultados obtenidos para cada curso.

Tabla 2: Análisis Curricular

\begin{tabular}{|c|c|c|c|c|c|}
\hline \multirow{2}{*}{$\begin{array}{l}\text { Bloques de } \\
\text { Análisis } \\
\text { Currículum }\end{array}$} & \multirow[b]{2}{*}{$\begin{array}{l}\text { Códigos de Contenidos de los Escenarios de } \\
\text { Futuro }\end{array}$} & \multicolumn{4}{|c|}{ Citas por cursos } \\
\hline & & $\begin{array}{c}1^{\circ} \\
\text { E.S.O. }\end{array}$ & $\begin{array}{c}2^{\circ} \\
\text { E.S.O. }\end{array}$ & $\begin{array}{c}3^{\circ} \\
\text { E.S.O. }\end{array}$ & $\begin{array}{l}4^{\circ} \\
\text { E.S.O. }\end{array}$ \\
\hline $\begin{array}{l}\text { Contaminación } \\
\text { General }\end{array}$ & Contaminación & 7 & 5 & & 4 \\
\hline Atmósfera & $\begin{array}{l}\text { Contaminación Atmosférica, Disminución } \\
\text { Contaminación Atm }\end{array}$ & 1 & 1 & 3 & 1 \\
\hline Agua & Impacto Acumulación de residuos, falta de agua & & 1 & & \\
\hline Suelo & Contaminación del Suelo & & & & 1 \\
\hline Biodiversidad & $\begin{array}{l}\text { Pérdida de Biodiversidad, Deforestación, } \\
\text { Destrucción del entorno natural, pérdida de } \\
\text { ecosistemas, pérdida de nuevas especies }\end{array}$ & 4 & 4 & 3 & 7 \\
\hline $\begin{array}{l}\text { Explotación de } \\
\text { recursos }\end{array}$ & $\begin{array}{l}\text { Disminución de recursos y agotamiento de } \\
\text { combustibles fósiles }\end{array}$ & 1 & 5 & 7 & 4 \\
\hline $\begin{array}{l}\text { Energías } \\
\text { Renovables }\end{array}$ & Energías alternativas & & 3 & 1 & \\
\hline Energía Nuclear & $\begin{array}{l}\text { Acumulación de Residuos Nucleares, alteraciones } \\
\text { genéticas }\end{array}$ & & & 1 & \\
\hline $\begin{array}{l}\text { Gestión de } \\
\text { residuos }\end{array}$ & Residuos Sólidos Urbanos e Industriales & 1 & & 1 & 1 \\
\hline $\begin{array}{l}\text { Calentamiento } \\
\text { Global }\end{array}$ & Calentamiento Global, cambio climático & 2 & & 4 & 4 \\
\hline $\begin{array}{l}\text { Análisis crítico } \\
\text { de Efectos } \\
\text { globales }\end{array}$ & $\begin{array}{l}\text { Crisis, Obesidad, sobrepoblación, Guerras, falta } \\
\text { de alimentos, pobreza, desequilibrios } \\
\text { internacionales, hambre, explotación infantil, } \\
\text { sedentarismo, emigración, conflictos armados, } \\
\text { economía insostenible, inmigración, crisis } \\
\text { políticas, tensiones políticas, emisiones por } \\
\text { transportes }\end{array}$ & 4 & 22 & 11 & 15 \\
\hline $\begin{array}{l}\text { Conductas y } \\
\text { Políticas } \\
\text { solidarias a } \\
\text { adoptar }\end{array}$ & $\begin{array}{l}\text { Reutilización de productos, desarrollo sostenible, } \\
\text { Derechos humanos, concienciación ambiental, } \\
\text { Educación Ambiental, igualdad, } \\
\text { solidaridad/salida crisis, ahorro de recursos }\end{array}$ & & 1 & 1 & 4 \\
\hline $\begin{array}{l}\text { Impacto del } \\
\text { desarrollo } \\
\text { tecnológico }\end{array}$ & $\begin{array}{l}\text { Usos de la energía eléctrica, desarrollo } \\
\text { tecnológico insostenible, accidentes de tráfico, } \\
\text { deterioro del medio natural, consumo compulsivo, } \\
\text { accidentalidad/inseguridad, disminución pobreza, } \\
\text { fin del hambre, espacio como nuevo hábitat, } \\
\text { aumento de ecosistemas y nuevas especies }\end{array}$ & & 1 & 3 & 5 \\
\hline \multicolumn{6}{|l|}{$\begin{array}{l}\text { Fabricación y } \\
\text { reciclaje }\end{array}$} \\
\hline \multicolumn{6}{|l|}{$\begin{array}{l}\text { Transgénicos y } \\
\text { biotecnología }\end{array}$} \\
\hline \multicolumn{6}{|l|}{$\begin{array}{l}\text { Plagas y control } \\
\text { biológico }\end{array}$} \\
\hline $\begin{array}{l}\text { Riesgos } \\
\text { Naturales }\end{array}$ & Ciclos naturales, catástrofes naturales & 1 & 1 & & \\
\hline & TOTAL CITAS & 21 & 44 & 35 & 46 \\
\hline
\end{tabular}

Fuente: Propia 


\section{$1^{o}$ E.S.O}

En este nivel educativo, salvo los temas relacionados con las energías, riesgos climáticos e importancia y conservación del agua dulce, todos los demás son mencionados por el alumnado. La figura 2 presenta la nube de palabras, resultado de volcar la tabla 2 - columna $1^{\circ}$ E.S.O. en la aplicación Wordle-Create. Como se puede observar las palabras de mayor tamaño de fuente coinciden con aquellas que presentan un mayor número de citas (frecuencia), en este caso la contaminación.

En un segundo plano la pérdida de biodiversidad presenta una relevancia notable, mientras que problemas globales como el calentamiento global o las guerras en consecuencia de la globalización estarían en el tercer lugar de importancia en cuanto a citas realizadas por el alumnado de $1^{\circ}$ E.S.O. Por su parte, la contaminación atmosférica en concreto, los residuos, la sobrepoblación aparecen en pocas ocasiones junto a los riesgos de la salud y pérdidas de ecosistemas asociadas a las problemáticas anteriores.

Figura 2: Nube de palabras sobre los temas de Educación Ambiental que aparecen en las C.E.F. en $1^{\circ}$ E.S.O.

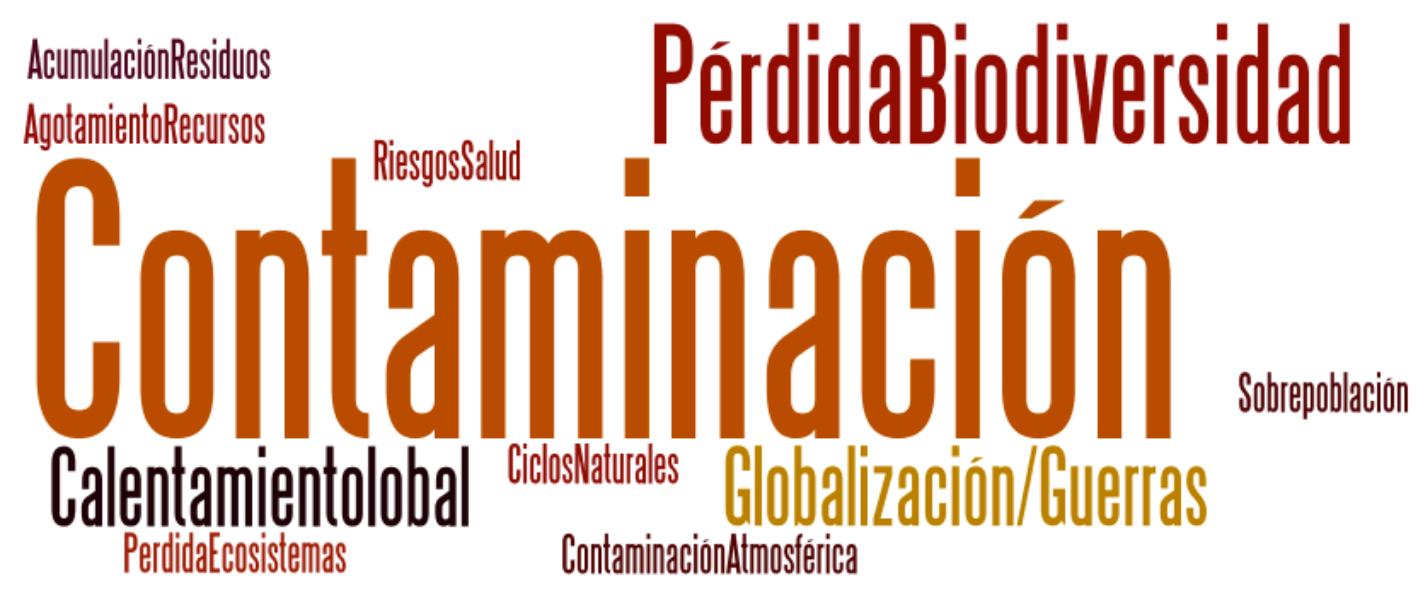

Fuente: Propia

Como resultado final, cabe destacar que el 38,7\% incorpora en sus escritos una relación directa con aspectos ambientales propios del curriculum de $1^{\circ}$ E.S.O., aunque no se ha tenido en cuenta las citas realizadas sobre otras cuestiones relacionadas con la Educación Ambiental. Con carácter general, es necesario indicar que el código "contaminación" se ha utilizado cuando en el análisis de contenido de las unidades semánticas se insertaba la palabra "contaminación" aunque no discriminaba si ésta era atmosférica, hídrica, o terrestre.

$2^{o}$ E.S.O

Como se observa en la tabla 2, la mayoría de los contenidos curriculares quedan reflejados en las C.E.F. salvo los relativos al papel protector de la atmósfera, los riesgos climáticos, las repercusiones de la energía eléctrica y el ahorro energético. Los 
resultados de la tabla 2 muestran que los temas propios del curriculum asociados directamente con el entorno natural, que más ha interiorizado el alumnado, son los relacionados con la Contaminación y Agotamiento de recursos (5 citas) y Agotamiento de recursos (4 citas). Destaca que en este grupo de $2^{\circ}$ la problemática ambiental con fuerte impacto social (Crisis, Derechos Humanos, Guerras, etc..) son las que presentan un mayor número de citas como lo demuestra las menciones a la crisis global que estamos viviendo, con 8 citas de las 22 que forman el total de esa temática.

Figura 3: Nube de palabras sobre los temas de Educación Ambiental que aparecen en las C.E.F. en $2^{\circ}$ E.S.O.

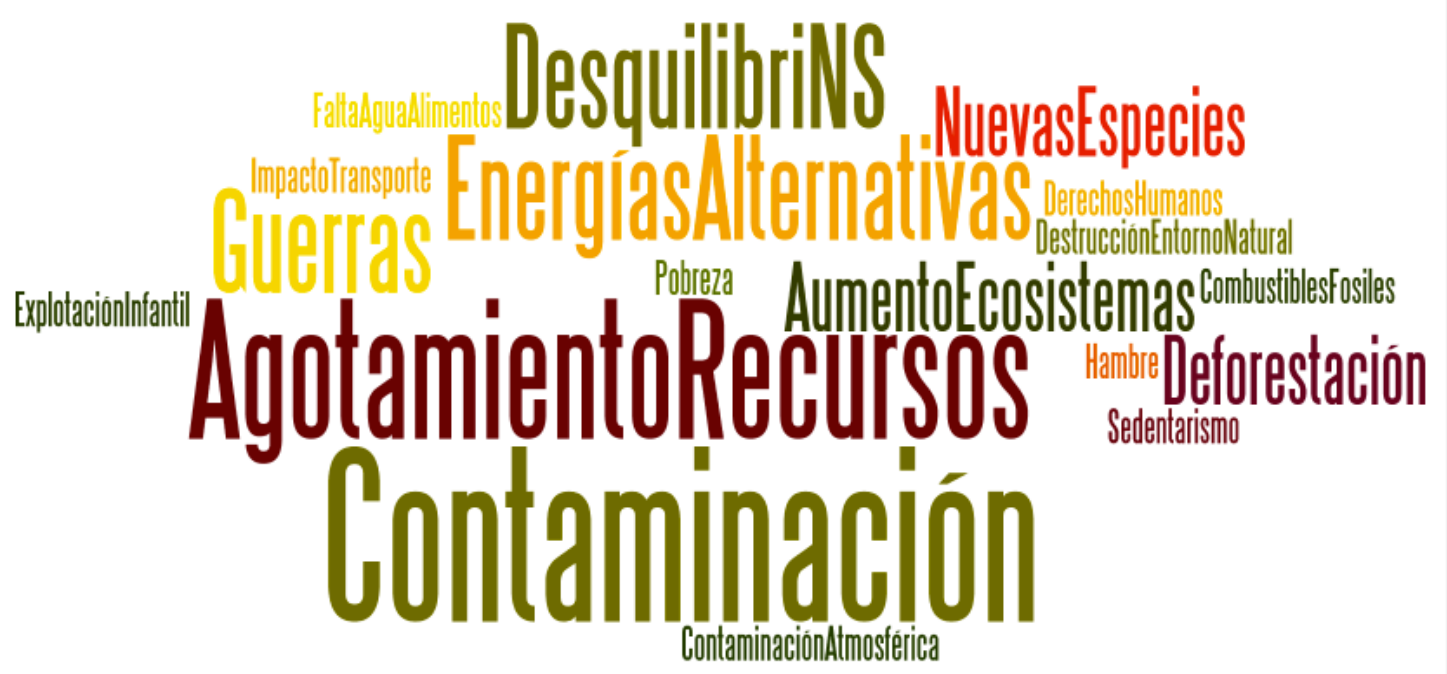

Fuente: Propia

Al realizar la nube de palabras (figura 3) se observan cuatro niveles de importancia en cuanto a nivel de citas se refiere. El primer nivel de interés está formado por la contaminación en general y el agotamiento de recursos y materias primas. En un segundo nivel se encuentran los desequilibrios Norte/Sur, el uso de energías alternativas, y las guerras. En el tercer nivel un reducido número de citas muestran cuestiones como la posibilidad de encontrar nuevas especies gracias a los avances tecnológicos, por el contrario en este nivel también se observa la deforestación como un problema.

Por último, en el cuarto nivel, los problemas como el hambre, el sedentarismo, la pobreza, la explotación infantil, los derechos humanos, aparecen ocasionalmente en alguna de las producciones escritas junto a otros problemas como la destrucción del entorno natural, el impacto del transporte, el uso y agotamiento de combustibles fósiles, y la falta de agua y alimentos. 


\section{$3^{\circ}$ E.S.O}

En este nivel se observa que los temas asociados al curriculum que más aparecen son los relacionados con el Agotamiento de recursos (8 citas) y con la Crisis y las cuestiones sociales de desarrollo sostenible y conflictos y desequilibrios sociales que suman 11 citas entre todas estas cuestiones lo que se refleja en la figura 4.

Figura 4: Nube de palabras sobre los temas de Educación Ambiental que aparecen en las C.E.F. en $3^{\circ}$ E.S.O.

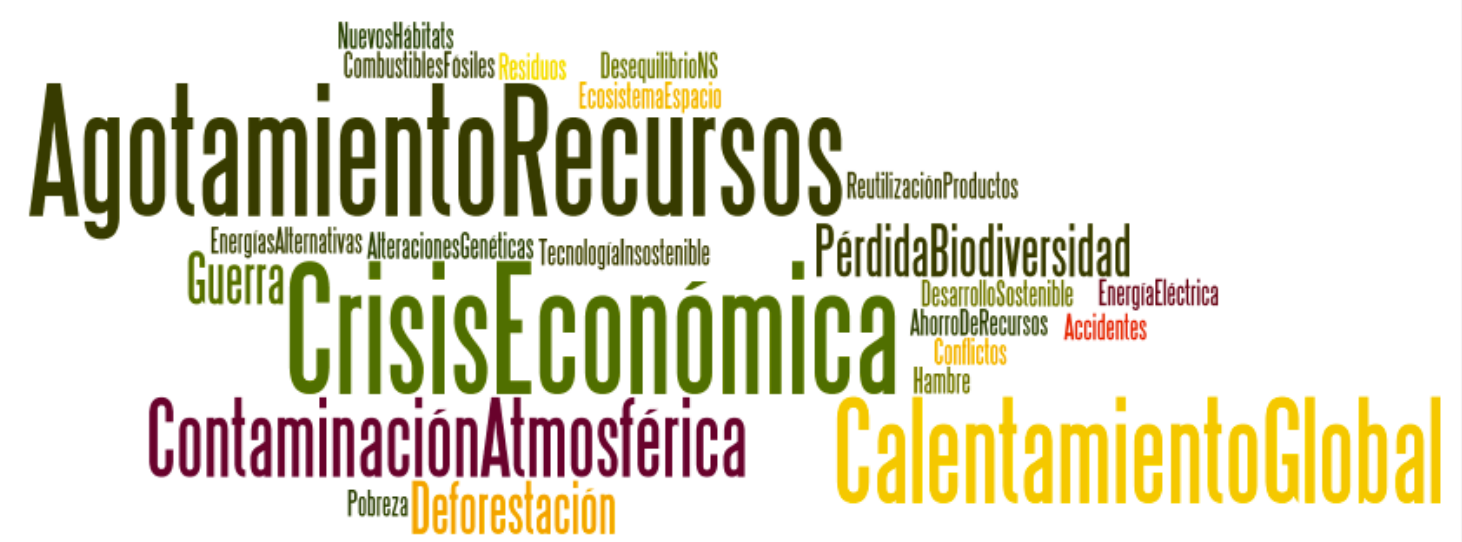

Fuente: Propia

Se observan cuatro niveles de importancia según la cantidad de citas analizadas en las producciones escritas de $3^{\circ}$ E.S.O. En el primer nivel, el agotamiento de recursos, la crisis económica, y el calentamiento global son las cuestiones más interiorizadas por la clase. En el segundo nivel se encontraría la contaminación atmosférica, y en el tercero la pérdida de biodiversidad, la deforestación y la guerra.

Por último en el cuarto nivel con un número de citas muy escaso, aparecen problemas sociales y globales como el hambre, los desequilibrios internacionales, la pobreza, el desarrollo sostenible, junto a ellos aparecen problemas asociados al uso de la energía eléctrica y los combustibles fósiles como el petróleo, y la ayuda que la tecnología podría prestar para la colonización de nuevos hábitats en el espacio y con ello nuevos ecosistemas, las energías alternativas, la reutilización de productos y la reutilización de recursos.

$4^{o}$ E.S.O

En la tabla 2, se puede observar como los temas relacionados con la Biodiversidad (7 citas) y los Impactos Tecnológicos (5 citas) son los más citados siempre por detrás de los impactos globales con alto contenido social (15 citas) 
Figura 5: Nube de palabras sobre los temas de Educación Ambiental que aparecen en las C.E.F. en $4^{\circ}$ E.S.O

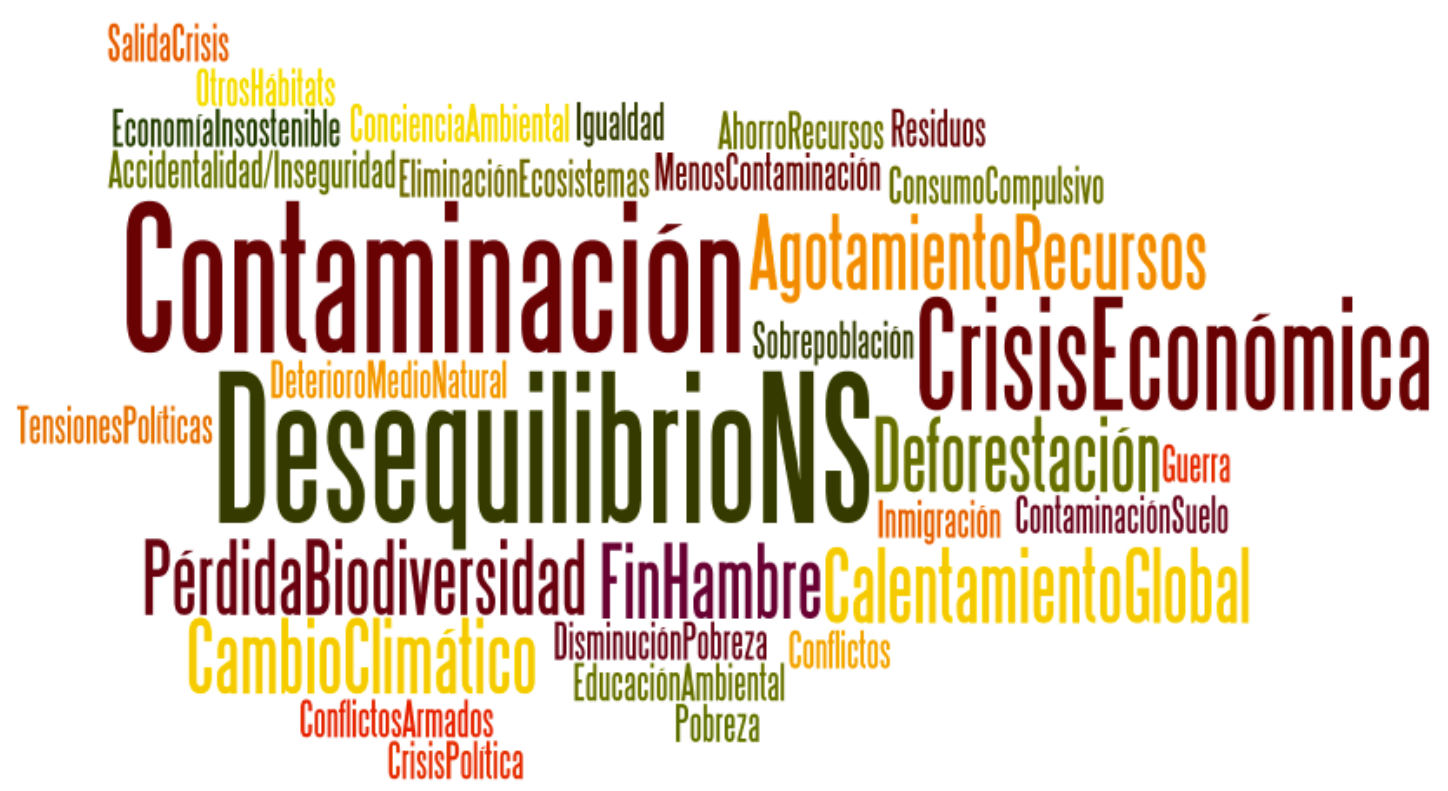

Fuente: Propia

La figura 5 , nube de palabras generada para el último curso de estudio ( $4^{\circ}$ E.S.O.), presenta una gran variedad de temática tratada. Cabe destacar que la dimensión social de la Educación Ambiental es la más tratada, cuestionando el sistema políticoeconómico predominante en Europa y el mundo desarrollado y sus implicaciones en la sostenibilidad tanto del planeta como en las relaciones sociales de la humanidad. A partir de lo anterior se pueden construir cuatro niveles de importancia según la cantidad de citas analizadas en las producciones escritas.

En el primer nivel se encuentra el número más elevado de citas que hacen referencia a la contaminación general y los desequilibrios sociales entre el hemisferio norte y el sur por la redistribución de la riqueza y la extracción de recursos. En el segundo nivel aparece un número destacable de citas que hacen referencia a la crisis económica como una cuestión preocupante para un gran número de estudiantes. En el tercer nivel se encuentran citas diseminadas por los textos producidos por los estudiantes que hacen referencia a la pérdida de biodiversidad, el calentamiento global, el agotamiento de recursos, el cambio climático, la deforestación, y un impacto positivo a nivel tecnológico como es el posible fin del hambre en el mundo.

Por último en el cuarto nivel, se incluyen citas que aparecen en algún texto de manera muy aislada observándose una gran cantidad de referencias a problemas tales como las guerras, las crisis políticas, la pobreza, las tensiones políticas, la inmigración, el consumo compulsivo, inseguridad, el tratamiento de residuos, la eliminación completa de ecosistemas. Frente a esto en algunos textos se observan citas que hacer referencia a la salida de la crisis global, soluciones tecnológicas y políticas frente al 
hambre, una economía sostenible, aumento de igualdad o la disminución del uso de recursos naturales.

\section{Discusión}

Para conocer si a través de la Construcción de Escenarios de Futuro el alumnado había adquirido los conocimientos propios de la Educación Ambiental del currículum, se procedió a localizar y codificar los conceptos utilizados por el alumnado, de acuerdo a la metodología ya explicada. Mediante los Escenarios de Futuro se aprecia que el $83,33 \%$ muestra en sus escritos una relación directa con aspectos ambientales propios del currículum de toda la E.S.O. sin tener en cuenta las citas realizadas sobre otras cuestiones relacionadas con la Educación Ambiental.

Por otro lado, el 46,88\% del alumnado muestra en sus escritos una relación directa con aspectos ambientales propios del entorno natural, dentro del recorrido curricular de $1^{\circ}$ y $2^{\circ}$ E.S.O. Si se tienen en cuenta las citas relacionadas con la crisis y las cuestiones como el hambre, guerra, desequilibrios entre países, etc... se llegaría al entorno del $85 \%$ del alumnado involucrado con temática relacionada con la Educación Ambiental. Por otro lado, $72,73 \%$ tiene una relación directa con aspectos ambientales propios del currículum desde $1^{\circ}$ E.S.O. a $3^{\circ}$ E.S.O. sin tener en cuenta las citas realizadas sobre otras cuestiones relacionadas con la Educación Ambiental.

Estos resultados parecen estar en consonancia con los análisis realizados por Kong (2014) donde se apreciaba que la mayoría del alumnado predecía alcanzar un correcto bienestar ambiental de manera prioritaria a través de la superación de los espacios de crisis económica o social. Además de acuerdo con el análisis de Kong en Barcelona y Santiago de Chile, se obtuvieron también porcentajes similares en cuanto a la aparición de problemáticas globales como la contaminación y calentamiento global. Sin embargo, no hemos encontrado ningún estudio que utilice la C.E.F. para determinar el porcentaje de contenidos en materia de educación ambiental asimilados por el alumnado. Por lo que no es posible establecer comparaciones.

\section{Conclusiones}

Podemos afirmar que la mayor parte de los contenidos de Educación ambiental fueron adquiridos por el alumnado. Sin embargo, cabe destacar la ausencia de cuestiones relacionadas con Riesgos Climáticos, y el Ahorro Energético, así como el papel Protector de la Atmósfera en los cursos de $1^{\circ}$ y $2^{\circ}$. Aunque posteriormente se refuerzan y aparecen como más trabajados por el alumnado sobre todo en $4^{\circ}$ de E.S.O.

Por el contrario, en $4^{\mathrm{o}}$ de E.S.O. dejan de aparecer cuestiones relativas a las Energías Alternativas, Reciclaje, o Biotecnología centrándose en el desarrollo de toda la problemática socio-política que rodea al medioambiente en su dimensión de relaciones interpersonales y salud pública. 
Durante la etapa de la E.S.O., la Educación Ambiental en los cuatro grupos analizados, con los condicionantes propios del estudio, resulta trabajada desde el punto de vista de la asimilación de contenidos curriculares. Se observa que se crea en ellos conciencia de los problemas existentes y soluciones posibles a nivel de grupo clase. Sin embargo, es preocupante el pesimismo que muestra el alumnado conforme va creciendo, y parece que la visión transmitida no genera en él una visión positiva del mundo y del futuro.

Conviene comentar que en general los aspectos relacionados con el agotamiento de recursos y la dimensión social de la Educación Ambiental son los que quedan más patentes en los escritos del alumnado.

Las C.E.F. analizadas en este trabajo han permitido ver como los estudiantes de E.S.O. están claramente preocupados por las implicaciones globales y sociales de las actuaciones que el ser humano está llevando a cabo en el planeta. A raíz del interés mostrado la C.E.F. puede resultar una herramienta docente eficaz para la determinación y programación de Proyectos Ambientales o incluso interdisciplinares destinados a modificar y mejorar las condiciones de la realidad inmediata en la que el alumnado está viviendo.

Coincidimos con Rivas, Herrera y Kushner (2009), cuando indican que el interés por las biografías no es, por tanto, individualista, centrado en el sujeto. Resulta más interesante comprender mejor la sociedad en que vivimos a partir de la actuación de cada uno y cada una de los que forman parte de ella. De este modo, si estos sujetos modifican su visión de la sociedad a partir de la reflexión sobre su propia vida, se están creando condiciones para transformar el mundo. Esta reflexión toma una gran importancia en relación con la Educación Ambiental, ya que traspuesta a esta área podríamos decir que: si los individuos de una sociedad reflexionan sobre las implicaciones que tienen sus acciones de la vida cotidiana sobre el planeta se están creando condiciones para la acción, para su participación en la resolución de los problemas ambientales.

La C.E.F. resultaría en ese momento una herramienta adecuada para valorar si las proyecciones de los futuros esperados van variando con el tiempo y acercándose a los futuros deseables por el alumnado, convirtiendo la escuela no solo en un centro de aprendizaje, sino de transformación de la realidad en el corto plazo, entendiendo el corto plazo un periodo de 10 años, momento en el que el alumnado que actualmente cursa la E.S.O. ya habrá cumplido la mayoría de edad, pudiendo ejercer entonces derechos participativos ciudadanos propios de una sociedad democrática. Además un periodo de 10 años parece asumible por ser práctica habitual en la planificación como por ejemplo la década por la educación por un futuro sostenible que se planteó de 2005 a 2014.

La experiencia personal docente dentro del ámbito de las Ciencias Experimentales y las Matemáticas en la E.S.O., nos hace pensar que la "capacidad de 
decisión del alumnado" es un aspecto de gran importancia a desarrollar en el trabajo con adolescentes. De nada sirve todo lo anterior si los estudiantes no son capaces o no tienen la posibilidad de estructurar su currículum y actuaciones desde el conocimiento construido en el aula haciendo uso de su libertad y su poder. Es aquí donde radica el mayor problema de la C.E.F., puesto que ley educativa y gran parte de las metodologías utilizadas a nivel de aula, encorsetan lo aprendido, de manera que la relación currículum-estudiante se produce desde el "vamos a estudiar esto" que "te servirá para". Creemos que la C.E.F. debería hacer cambiar esta dirección de aprendizaje de modo que la relación currículum-estudiante se transforme en un "para cambiar el futuro necesito saber esto" por lo que "vamos a estudiarlo para aprender y cambiar mi entorno". Lo anterior no solo precisaría de un currículum más abierto, sino de un profesorado capaz de orientar y canalizar las inquietudes de sus estudiantes ante cuestiones que les pudieran pasar desapercibidas o poco relevantes y que realmente fueran a ser necesarias en su vida cotidiana posterior, puesto que como ya se ha comentado los adolescentes presentan una visión cortoplacista.

Con todo lo anterior en futuras investigaciones, se podrían utilizar muestras mucho más amplías, así como contextos socio-económicos diversos con objeto de realizar comparativas de resultados. Además, la técnica de narrativa individual se podría triangular con otros métodos como los focus-groups o grupos de discusión, y otras técnicas como las plásticas.

\section{Referencias}

Álvarez-Gayou, J.L. (2003). Cómo hacer investigación cualitativa: fundamentos y metodología. México: Paidós

Calderón, I. y Garrido, G. (2002), Metodologías agresivas, alumnos violentos. Reflexiones desde un estudio de caso. Revista Interuniversitaria de Formación del Profesorado, 5(4). Recuperado de https://dialnet.unirioja.es/descarga/articulo/1034338.pdf

Erickson, F. (1986). Qualitative methods in research on teaching. En M.C. Wittrock (Ed). Handbook of research on teaching (Third Edition). New York: Macmillan.

Hernández, F. (2004). Las historias de vida como estrategia de visibilización y generación de saber pedagógico. En: Goodson, Ivor, F. (Ed.), Historias de vida del profesorado. Barcelona: Octaedro - EU B.

Jiménez Van der Biest, T. (2006).La narración escrita infantil: Un estudio en niños de educación básica. Revista de Investigación, 60, 157-174.

Khan, H. y Wiener, A (1967). The year 200, a framework for speculation on the next thirty-tree years. Washington: The Hudson Institute. 
Kleiner, A. (1999) Doing Scenarios - scenarios can help predict the future. Formerly Whole Earth Review (W. Earth, Ed.) Invierno

Kong, F. (2014). Construcción de Escenarios de Futuro como aportación Didáctica y Metodológica para una Educación Ambiental creativa, Global y sostenible. Tesis doctoral sin publicar. Universidad de Barcelona.

López-Aranguren, E. (2015). El análisis de contenido tradicional. En M. García Ferrando, F.R. Alvira, L.E. Alonso y R. Modesto (Coords), El análisis de la realidad social: métodos y técnicas de investigación. Madrid: Alianza.

Muñoz, J.M. (2010). La Educación Ambiental como eje transversal en el currículo. Revista Innovación y Experiencia Educativas, 29, 1-9.

Novo, M. (2006). Educación Ambiental. Desarrollo sostenible y globalización. Revista de Educación Ambiental, 4 (6), 24-31.

Pascual, J. A., Molina, J., Martínez, R., Curiel, E., y Ramírez, E. (2000). La integración de la Educación Ambiental en la E.S.O. Enseñanza de las Ciencias, 18(2), 227234.

Polkinghorne, D. (1995). Narrative configuration in qualitative analysis. En Hatch, J., y Wisniewski, R. (Eds), Life history and narrative. Londres: Falmer press.

Porter, M. (2004). Competitive Advantage: creating and sustaining superior performance. New York: New Ed Edition.

Reparaz, CH., y Naval, C. (2012). Ilusiones y miedos de los niños ante el futuro. Informes y Estudios (Institución Futuro), N. 12, 2007

Rivas, I., Herrera, D. Y Kushner, S. (2009). Voz y educación: La narrativa como enfoque de interpretación de la realidad. Barcelona. Octaedro.

Schwartz, P. (1996). The Art of the Long View: Planning for the Future in an Uncertain World. New York: Currency Doubleday.

Sessano, P. (2006). La Educación Ambiental: un modo de aprender. Anales de la educación común, 2(3), 102-11.

Suárez, M. (2002), Historias de vida y fuente oral. Los recuerdos escolares. En: Escolano, Agustín y Hernández, J. M. (Coords.), La memoria y el deseo. Cultura de la escuela y educación deseada. Valladolid: Tirant lo Blanch. (pp. 107-133).

Suárez, M. (2004), Los castigos y otras estrategias disciplinarias vistos a través de los recuerdos escolares. Revista de Educación, 335, 429-443.

UNESCO (2006). Plan de aplicación Internacional del Decenio de las Naciones Unidas de Educación para el Desarrollo Sostenible. Paris: Sección Educación.

Vergara, J.C., Fontalvo T.J. y Maza, F. (2010). La planeación por escenarios. Revisión de conceptos y propuestas metodológicas. Prospective, 8(2), 21-29. 
Wack, P. (1985). Scenarios: Uncharted Water Ahead. Harvard Business Review, 63(5), 73-89.

Fecha de recepción: 06/08/2016

Fecha de revisión: 27/10/2016

Fecha de aceptación: 23/11/21016 
